\title{
Dependence of Photoinduced Bending Behavior of Diarylethene Crystals on Ultraviolet Irradiation Power
}

Akira Hirano, Takuya Hashimoto, Daichi Kitagawa, Kenji Kono, Seiya Kobatake

\begin{tabular}{|c|l|}
\hline Citation & Crystal Growth \& Design, 17 (9); 4819-4825 \\
\hline Issue Date & $2017-08-03$ \\
\hline Type & Journal Article \\
\hline Textversion & author \\
\hline $\begin{array}{c}\text { Supporting } \\
\text { Information }\end{array}$ & $\begin{array}{l}\text { Supporting Information is available free of charge on the ACS Publications website at } \\
\text { https://doi.org/10.1021/acs.cgd.7b00755. }\end{array}$ \\
\hline Rights & $\begin{array}{l}\text { This document is the Accepted Manuscript version of a Published Work that appeared } \\
\text { in final form in Crystal Growth \& Design, copyright } @ \text { American Chemical Society } \\
\text { after peer review and technical editing by the publisher. To access the final edited and } \\
\text { published work see https://doi.org/10.1021/acs.cgd.7b00755 . }\end{array}$ \\
\hline DOI & \begin{tabular}{l}
$10.1021 /$ acs.cgd.7b00755 \\
\hline
\end{tabular}
\end{tabular}

\author{
Self-Archiving by Author(s) \\ Placed on: Osaka City University
}




\section{Dependence of Photoinduced Bending Behavior of Diarylethene Crystals on Ultraviolet Irradiation}

\section{Power}

Akira Hirano,,$^{\dagger}$ Takuya Hashimoto, ${ }^{\dagger}$ Daichi Kitagawa, ${ }^{\dagger}$ Kenji Kono, ${ }^{*}$ and Seiya Kobatake* ${ }^{\dagger}$

${ }^{\dagger}$ Department of Applied Chemistry, Graduate School of Engineering, Osaka City University, 3-

3-138 Sugimoto, Sumiyoshi-ku, Osaka 558-8585, Japan

Department of Applied Chemistry, Graduate School of Engineering, Osaka Prefecture

University, 1-1 Gakuen-cho, Naka-ku, Sakai, Osaka 599-8531, Japan

KEYWORDS: Photochromism; diarylethene; photomechanical crystal; bending; light power 
ABSTRACT: The photoinduced bending behavior of diarylethene crystals upon irradiation with various ultraviolet (UV) light intensities was investigated. The bending velocity of the photoinduced bending crystals was estimated from the curvature of the bending crystal. The initial velocity of curvature change ( $\left.V_{\text {init }}\right)$ increased in proportion to the power of the incident UV light even when the crystal thickness was different, which suggests that the local strain caused by photoisomerization makes a cumulative contribution to the bending behavior. Moreover, for all UV light intensities, the relationship between $V_{\text {init }}$ and the crystal thickness was well explained by the easily handled Timoshenko bimetal model. This result provides a validated method for the quantitative evaluation of the photoinduced bending velocity in various molecular crystals. 


\section{INTRODUCTION}

Diarylethenes are well known as photochromic compounds that can reversibly change their chemical and physical properties upon alternating irradiation with UV and visible light, and can undergo photochromic reactions even in polymer films and crystalline phases as well as in solution. ${ }^{1,2}$ Diarylethene crystals exhibit various photomechanical motions such as contraction, ${ }^{3,4}$ expansion, ${ }^{5}$ bending, ${ }^{3,5-12}$ separation, ${ }^{13}$ rolling, ${ }^{6}$ and twisting. ${ }^{14}$ Other photochromic compounds that exhibit photomechanical motion in their crystal state, such as furylfulgides, ${ }^{15}$ azobenzenes, ${ }^{16,17}$ salicylideneanilines, ${ }^{18}$ and anthracene carboxylates ${ }^{19-24}$ have been reported. Among these photochromic compounds, diarylethene derivatives are the most promising compounds that function as photomechanical actuators because of their rapid response, fatigue resistance, and thermal stability. ${ }^{1,2}$ Photomechanical motion has been attracting much attention because such motion enables the direct conversion of light energy to mechanical energy by an external stimuli, and without contact or electric wires. In particular, bending behavior is the most promising motion for photomechanical actuators. Thus, it is essential to investigate crystal bending behavior in more detail for the development of the photomechanical crystals.

There are two types of bending direction in photoinduced crystal bending, bending away from ultraviolet (UV) light and bending toward UV light, which occur due to expansion and contraction along the long axis of the photoreacted crystal layer, respectively. In both types, photoinduced crystal bending is caused by heterogeneous and gradient photoconversion with respect to depth because of the high absorption of the crystal in the UV region. In our previous work, we have reported the dependence of the photoinduced bending behavior of diarylethene crystals on the crystal thickness. ${ }^{5,9}$ Thin crystals bend rapidly and to a greater extent. To quantitatively discuss the bending behavior, we have introduced the Timoshenko equation, which is known as the 
simplified bimetal model. We have succeeded in explaining the correlation between the initial velocity of the curvature change and the crystal thickness by the Timoshenko bimetal model in both types, expansion and contraction, of the photoreacted crystal surface. However, this method has a drawback in that the experimental error may be large because the bending behavior was observed only at a certain intensity of UV light.

Herein, we report the dependence of the photoinduced bending behavior of diarylethenes 1-3 (Scheme 1) on the power of the incident UV light to reveal whether local strain acts cumulatively due to the structural changes of individual diarylethene molecules or cooperatively due to a phase transition or the formation of domains in the crystal.

\section{[Scheme 1]}




\section{RESULTS AND DISCUSSION}

In all diarylethenes $\mathbf{1}-\mathbf{3}$, their rod-like crystals can be crystallized by slow evaporation of the solvents. X-ray crystallographic data are summarized in Table S1. Diarylethene molecules in all crystals of 1-3 are fixed in an antiparallel conformation and the distance between the reactive carbons is shorter than $4.2 \AA$, which is sufficiently short for photoisomerization from the openring isomer to the closed-ring isomer to occur in the crystalline phase. ${ }^{25} \mathrm{UV}$ light irradiation was

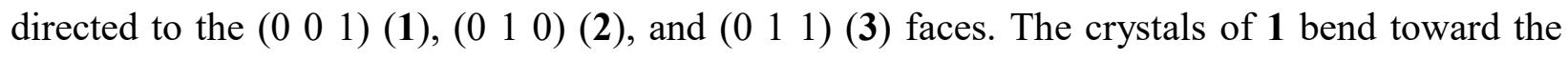
incident light, whereas crystals $\mathbf{2}$ and $\mathbf{3}$ bend away from the incident light, as shown in Figure 1. Photoisomerization of diarylethene molecules from the open-ring isomer to the closed-ring isomer upon UV irradiation causes contraction or expansion along the long axis of the crystal, which results in bending of the crystal. The bent crystals can then return to their initial straight shape upon irradiation with visible light.

Figure 2 shows the crystal shapes and molecular packing diagrams for crystals $\mathbf{1}, \mathbf{2}$, and $\mathbf{3}$. The crystal systems and space groups of $\mathbf{1}, \mathbf{2}$, and $\mathbf{3}$ are monoclinic $P 2_{1} / \mathrm{n}$, triclinic $P \overline{1}$, and monoclinic $P 2_{1} / \mathrm{c}$, respectively. Crystal 1 shows that the molecules have herringbone type molecular packing, whereas crystals $\mathbf{2}$ and $\mathbf{3}$ do not have herringbone type molecular packing. Diarylethene crystals with herringbone type packing tend to exhibit crystal contraction by photocyclization; $;^{3,4,12,26}$ however, a more detailed study of the correlation between molecular packing and the bending direction is required.

\section{[Figure 1]}

[Figure 2] 
Figure 3 shows the changes in the curvature of crystals 1, 2, and $\mathbf{3}$ irradiated with various UV light intensities. The crystal thicknesses of crystals $\mathbf{1}, \mathbf{2}$, and $\mathbf{3}$ are $7.3,6.7$, and $2.7 \mu \mathrm{m}$, respectively. The rod-like crystal was mounted on a glass capillary, and a fluorescent material was painted on the glass capillary to observe the onset of UV irradiation. Observations were conducted using a CCD video camera attached to a digital microscope with a frame rate of 28 fps. The irradiation time in Figures 3a, c, and e shows that 1 frame before the first frame with blue fluorescence on the glass capillary is set to $0 \mathrm{~s}$. Therefore, the actual onset time is between 0 and $1 / 28 \mathrm{~s}$. The curvature is significantly increased with the power of the UV light. To quantitatively evaluate the effect of the UV light power on the photoinduced bending velocity, the initial velocity of curvature change ( $\left.V_{\text {init }}\right)$ was determined from the initial slope of the curvature change against the UV irradiation time. Figure 3 also shows plots of $V_{\text {init }}$ as a function of the irradiation power of the $\mathrm{UV}$ light. The $V_{\text {init }}$ value increased in proportion to the power of the incident UV light in all cases. Therefore, the photoinduced bending velocity of diarylethene crystals $\mathbf{1}-\mathbf{3}$ became larger in proportion to the power of the incident UV light.

\section{[Figure 3]}

Figure 4 shows plots of the curvature against the total energy of the incident light. Herein, the irradiation time was corrected using the actual onset time. The curvature gradually increased with the total energy of the incident light without dependence on the power of the incident light. Therefore, the curvature is dependent on photochromic conversion. The photocyclization reaction of diarylethene in the crystal state occurs by way of a one-photon absorption under irradiation power in the present experiment, and the possibility of a photoinduced phase transition during the photochromic reaction, photoinduced domain formation in the reactant area, or photoreaction by way of multiphoton absorption can all be excluded. Thus, the local strain that arises from the 
photoirradiated crystal acts cumulatively due to the structural changes of individual diarylethene molecules.

\section{[Figure 4]}

The $V_{\text {init }}$ values of diarylethene crystals with various crystal thicknesses were determined upon irradiation with various powers of UV light, as shown in Figure 5. In all cases, the $V_{\text {init }}$ value was proportional to the power of the irradiated UV light, even for different crystal thicknesses. These results indicate that $V_{\text {init }}$ is dependent on the conversion of diarylethene molecules by photoisomerization from the open-ring isomer to the closed-ring isomer and the local shape change of each diarylethene molecule induced by photochromic reaction on the crystal surface contributes cumulatively to the bending behavior.

\section{[Figure 5]}

We have reported the dependence of the photoinduced bending behavior on the crystal thickness. ${ }^{5,9}$ The relationship between $V_{\text {init }}$ and the crystal thickness could be well explained by the easily handled Timoshenko bimetal model. ${ }^{27}$ Naumov et al. and Bardeen et al. have independently

reported more sophisticated mathematical treatments based on a heterometry mechanism..$^{28-30}$ Such a rigorous analysis based on the experimental results is required for development of this research field. However, the quantitative argument of the bending velocities among different molecular crystals within an easily handled model is attractive. An illustration of the Timoshenko bimetal model and the corresponding equation are shown in Figure 6 and eq 1.

[Figure 6] 


$$
\begin{aligned}
\text { Curvature } & =\frac{1}{R}=\frac{\alpha_{2}-\alpha_{1}}{h_{2}} \frac{6 m n(1+m)}{1+4 m n+6 m^{2} n+4 m^{3} n+m^{4} n^{2}} \\
& =\frac{\alpha_{2}}{h_{2}} \frac{6 m(1+m)}{1+4 m+6 m^{2}+4 m^{3}+m^{4}}
\end{aligned}
$$

where $R$ is the curvature radius, $\alpha_{\mathrm{i}}(\mathrm{i}=1,2)$ are the actuation strains, $h_{\mathrm{i}}(\mathrm{i}=1,2)$ are the layer thicknesses, $m=h_{1} / h_{2}, n=E_{1} / E_{2}$, and $E_{\mathrm{i}}(\mathrm{i}=1,2)$ are the Young's moduli. As we have reported previously, the Timoshenko equation becomes quite simple through some assumptions, as shown in eq $2 .{ }^{26}$ In the initial stage of photoinduced bending, the correlation between the initial curvature change and the crystal thickness can be explained as eq 3 using $\alpha_{2}$,init, the initial velocity of $\alpha_{2}$. The values of $h_{2}$ and $\alpha_{2 \text {,init }}$ can be obtained by the best fitting curve (eq 3 ) to the experimental data.

$$
V_{\text {init }}=\frac{\alpha_{2, \text { init }}}{h_{2}} \frac{6 m(1+m)}{1+4 m+6 m^{2}+4 m^{3}+m^{4}}
$$

In previous work, the values of $h_{2}$ and $\alpha_{2}$,init were estimated by only fitting to experimental data obtained with UV light irradiation at a power of $55 \mathrm{~mW} \mathrm{~cm}^{-2}$. In the present work, we have confirmed that the value of $V_{\text {init }}$ is proportional to the power of the irradiated UV light. Therefore, when light with different power is used for irradiation, the $V_{\text {init }}$ value can be normalized by dividing it by the power of the light $(P)$. Table 1 shows the $V_{\text {init }} P^{-1}$ values determined from the slope in Figure 3, together with those determined from the $V_{\text {init }}$ and $P$ values in our previous paper. ${ }^{26}$ Figure 7 shows the relationship between $V_{\text {init }} P^{-1}$ and the crystal thickness. For all diarylethenes $\mathbf{1}-\mathbf{3}$, the experimental data were well fitted by the Timoshenko bimetal model. Table 2 shows the values of $h_{2}$ and $\alpha_{2, \text { init }} P^{-1}$ determined from the fitting curves shown in Figure 7 for diarylethenes $\mathbf{1}-\mathbf{3}$. The 
present data for diarylethenes $\mathbf{1}$ and $\mathbf{2}$ support our previous results. ${ }^{26}$ The data for diarylethene $\mathbf{3}$ were newly determined in the present work. The apparent bending velocity correlated with the irradiation power for 1-3 can be compared using $\alpha_{2, \text { init }} P^{-1}$. The velocities for $\mathbf{1}$ and $\mathbf{2}$ are not different from each other. However, the velocity of diarylethene $\mathbf{3}$ is slower than those of $\mathbf{1}$ and $\mathbf{2}$ by a factor of ca. 1.6.

\section{[Table 1]}

[Figure 7]

\section{[Table 2]}

Here we discuss the dependence of the $h_{2}$ value on the bending velocity. Although the bending velocity can be compared using the $\alpha_{2, \text { init }} P^{-1}$ values, the potential capacity of the bending cannot be evaluated from these values. For example, the $h_{2}$ value is dependent on the molar absorption coefficient at the irradiation wavelength; i.e., it is dependent on the molecular structure, ${ }^{26}$ irradiation wavelength, ${ }^{11}$ and irradiated crystal face. ${ }^{5}$ In our previous paper, we reported that the multiplication of $h_{2}$ and $\alpha_{2 \text {,init }}$ is constant when the same molecular structure, irradiation wavelength, and irradiation power are used. ${ }^{26}$ Therefore, the multiplication of $h_{2}$ and $\alpha_{2}$,init corresponds to the potential capacity of the bending. The values of $h_{2} \alpha_{2}$,init $P^{-1}$ are also shown in Table 2. The relative potential capacities of diarylethenes $\mathbf{1}-\mathbf{3}$ were 3.0,1.4, and 1.0, respectively. Therefore, the potential capacity of crystal $\mathbf{1}$ is the largest among these three compounds. We have thus succeeded in quantitative evaluation of the bending behavior, not only with respect to the crystal thickness and the value of $h_{2}$, but also with respect to the normalized irradiation power. 


\section{EXPERIMENTAL SECTION}

General. The photoinduced bending behavior of diarylethene crystals was observed using a Keyence VHX-500 digital microscope. UV irradiation was conducted using a Keyence UV-LED UV-400 with an attached UV-50H head (365-nm light). The light power on the crystal surface was measured using a Neoark PM-335A power meter. Visible light irradiation was conducted using a halogen lamp (100 W). Single-crystal X-ray crystallographic analysis was conducted using a Rigaku AFC/Mercury CCD diffractometer with Mo $K_{\alpha}$ radiation $(\lambda=0.71073 \AA)$ through a graphite monochromator. The crystal structures were solved by a direct method using SIR92 and refined by the full-matrix least-squares method on $F^{2}$ with anisotropic displacement parameters for non-hydrogen atoms using SHELXL-97.

Determination of the Initial Velocity of the Curvature Change. The edge of a rod-like crystal was fixed to a glass capillary and a fluorescent material was painted on the glass capillary. The fluorescence of the glass capillary shows the onset time of UV irradiation. The value of the curvature against UV irradiation time was plotted. The initial velocity of the curvature change was calculated from the initial slope of the curvature change.

Materials. Diarylethenes 1-3 were synthesized according to a procedure previously described in the literature. ${ }^{5,26,31}$

\section{CONCLUSIONS}

We have investigated the dependence of the photoinduced bending behavior of diarylethene crystals on the irradiation power of UV light. The initial velocity of curvature change was 
proportional to the power of the UV irradiation. These results suggested that local strain acts cumulatively due to the structural changes of individual diarylethene molecule at the initial stage of the photochromic reaction. Moreover, the correlation between the bending velocity and the crystal thickness can be well explained by the Timoshenko bimetal model. We have succeeded in quantitative evaluation of the capacity of photoinduced bending velocity among different diarylethene crystals using the thickness of the photoreacted layer $\left(h_{2}\right)$ in the bimetal model and the value of actuation strain $\left(\alpha_{2}\right.$,init $)$.

\section{ASSOCIATED CONTENT}

\section{Supporting Information}

Crystal data for diarylethenes 1-3 (Table S1) and crystallographic data of $\mathbf{3}$ in CIF format. This material is available free of charge via the Internet at http://pubs.acs.org.

\section{AUTHOR INFORMATION}

\section{Corresponding Author}

*E-mail: kobatake@a-chem.eng.osaka-cu.ac.jp

\section{Author Contributions}

The experimental work and data collection were conducted by A.H. and T.H. The work was discussed by all authors. The draft of the paper was written by D.K., K.K., and S.K. All authors have given approval to the final version of the manuscript. 


\section{Notes}

The authors declare no competing financial interest.

\section{ACKNOWLEDGMENT}

This work was partly supported by JSPS KAKENHI Grant Number JP26107013 in Scientific

Research on Innovative Areas "Photosynergetics" to S.K. and JSPS KAKENHI Grant Number JP16K17896 in Scientific Research for Young Scientists (B) to D.K. T.H. acknowledges

Program for Leading Graduate Schools for System-inspired Leaders in Material Science: SiMS, Osaka Prefecture University/Osaka City University from MEXT, Japan. The authors also thank Nippon Zeon Co., Ltd. for providing octafluorocyclopentene. 


\section{REFERENCES}

(1) Irie, M. Diarylethenes for memories and switches. Chem. Rev. 2000, 100, 1685-1716.

(2) Irie, M.; Fukaminato, T.; Matsuda, K.; Kobatake, S. Photochromism of diarylethene molecules and crystals: memories, switches, and actuators. Chem. Rev. 2014, 114, 12174-12277.

(3) Kobatake, S.; Takami, S.; Muto, H.; Ishikawa, T.; Irie, M. Rapid and reversible shape changes of molecular crystals on photoirradiation. Nature 2007, 446, 778-781.

(4) Kuroki, L.; Takami, S.; Yoza, K.; Morimoto, M.; Irie, M. Photoinduced shape changes of diarylethene single crystals: correlation between shape changes and molecular packing. Photochem. Photobiol. Sci. 2010, 9, 221-225.

(5) Kitagawa, D.; Kobatake, S. Crystal thickness dependence of photoinduced crystal bending of 1,2-bis(2-methyl-5-(4-(1-naphthoyloxymethyl)phenyl)-3-thienyl)perfluorocyclopentene. $\quad J$. Phys. Chem. C 2013, 117, 20887-20892.

(6) Uchida, K.; Sukata, S.; Matsuzawa, Y.; Akazawa, M.; de Jong, J. J. D.; Katsonis, N.; Kojima, Y.; Nakamura, S.; Areephong, J.; Meetsma, A.; Feringa, B. L. Photoresponsive rolling and bending of thin crystals of chiral diarylethenes. Chem. Commun. 2008, 326-328.

(7) Morimoto, M.; Irie, M. A diarylethene cocrystal that converts light into mechanical work. J. Am. Chem. Soc. 2010, 132, 14172-14178.

(8) Terao, F.; Morimoto, M.; Irie, M. Light-driven molecular-crystal actuators: rapid and reversible bending of rodlike mixed crystals of diarylethene derivatives. Angew. Chem. Int. Ed. 2012, 51, 901-904. 
(9) Kitagawa, D.; Kobatake, S. Crystal thickness dependence of the photoinduced crystal bending of 1-(5-methyl-2-(4-( $p$-vinylbenzoyloxymethyl)phenyl)-4-thiazolyl)-2-(5-methyl-2phenyl-4-thiazolyl)perfluorocyclopentene. Photochem. Photobiol. Sci. 2014, 13, 764-769.

(10) Kitagawa, D; Kobatake, S. Photoreversible current ON/OFF switching by the photoinduced bending of gold-coated diarylethene crystals. Chem. Commun. 2015, 51, 4421-4424.

(11) Kitagawa, D.; Tanaka, R.; Kobatake, S. Dependence of photoinduced bending behavior of diarylethene crystals on irradiation wavelength of ultraviolet light. Phys. Chem. Chem. Phys. 2015, $17,27300-27305$.

(12) Ohshima, S.; Morimoto, M.; Irie, M. Light-driven bending of diarylethene mixed crystals Chem. Sci. 2015, 6, 5746-5752.

(13) Kobatake, S.; Hasegawa, H.; Miyamura, K. High-convertible photochromism of a diarylethene single crystal accompanying the crystal shape deformation. Cryst. Growth Des. 2011, $11,1223-1229$.

(14) Kitagawa, D.; Nishi, H.; Kobatake, S. Photoinduced twisting of a photochromic diarylethene crystal. Angew. Chem. Int. Ed. 2013, 52, 9320-9322.

(15) Koshima, H.; Nakaya, H.; Uchimoto, H.; Ojima, N. Photomechanical motion of furylfulgide crystals. Chem. Lett. 2012, 41, 107-109.

(16) Koshima, H.; Ojima, N.; Uchimoto, H. Mechanical motion of azobenzene crystals upon photoirradiation. J. Am. Chem. Soc. 2009, 131, 6890-6891.

(17) Koshima, H.; Ojima, N. Photomechanical bending of 4-aminoazobenzene crystals. Dyes Pigm. 2012, 92, 798-801. 
(18) Koshima, H.; Takechi, K.; Uchimoto, H.; Shiro, M.; Hashizume, D. Photomechanical bending of salicylideneaniline crystals. Chem. Commun. 2011, 47, 11423.

(19) Al-Kaysi, R. O.; Müller, A. M.; Bardeen, C. J. Photochemically driven shape changes of crystalline organic nanorods. J. Am. Chem. Soc. 2006, 128, 15938-15939.

(20) Al-Kaysi, R. O; Bardeen, C. J. Reversible photoinduced shape changes of crystalline organic nanorods. Adv. Mater. 2007, 19, 1276-1280.

(21) Zhu, L.; Al-Kaysi, R. O.; Dillon, R. J.; Tham, F. S.; Bardeen, C. J. Crystal structures and photophysical properties of 9-anthracene carboxylic acid derivatives for photomechanical Applications. Cryst. Growth Des. 2011, 11, 4975-4983.

(22) Zhu, L.; Al-Kaysi, R. O.; Bardeen, C. J. Reversible photoinduced twisting of molecular crystal microribbons. J. Am. Chem. Soc. 2011, 133, 12569-12575.

(23) Zhu, L.; Agarwal, A.; Lai, J.; Al-Kaysi, R. O.; Tham, F. S.; Ghaddar, T.; Mueller, L.; Bardeen, C. J. Solid-state photochemical and photomechanical properties of molecular crystal nanorods composed of anthracene ester derivatives. J. Mater. Chem. 2011, 21, 6258-6268.

(24) Kim, T.; Al-Muhanna, M. K.; Al-Suwaidan, S. D.; Al-Kaysi, R. O.; Bardeen, C. J. Photoinduced curling of organic molecular crystal nanowires. Angew. Chem. Int. Ed. 2013, 52, 6889-6893.

(25) Kobatake, S.; Uchida, K.; Tsuchida, E.; Irie, M. Single-crystalline photochromism of diarylethenes: reactivity-structure relationship. Chem. Commun. 2002, 2804-2805.

(26) Kitagawa, D.; Iwaihara, C.; Nishi, H.; Kobatake, S. Quantitative evaluation of photoinduced bending speed of diarylethene crystals. Crystals 2015, 5, 551-561 
(27) Timoshenko, S. Analysis of bi-metal thermostats. J. Opt. Soc. Am. 1925, 11, 233-255.

(28) Nath, N. K.; Pejov, L.; Nichols, S. M.; Hu, C.; Saleh, N.; Kahr, B.; Naumov, P. Model for photoinduced bending of slender molecular crystals. J. Am. Chem. Soc. 2014, 136, 2757-2766.

(29) Kim, T.; Zhu, L.; Mueller, L. J.; Bardeen, C. J. Mechanism of photoinduced bending and twisting in crystalline microneedles and microribbons composed of 9-methylanthracene. J. Am. Chem. Soc. 2014, 136, 6617-6625.

(30) Naumov, P.; Chizhik, S.; Panda, M. K.; Nath, N. K.; Boldyreva, E. Mechanically responsive molecular crystals Chem. Rev. 2015, 115, 12440-12490.

(31) Browne, W. R.; de Jong, J. J. D.; Kudernac, T.; Walko, M.; Lucas, L. N.; Uchida, K.; van Esch, J. H.; Feringa, B. L. Oxidative electrochemical switching in dithienylcyclopentenes, Part 2: Effect of substitution and asymmetry on the efficiency and direction of molecular switching and redox stability. Chem. Eur. J. 2005, 11, 6430-6441. 

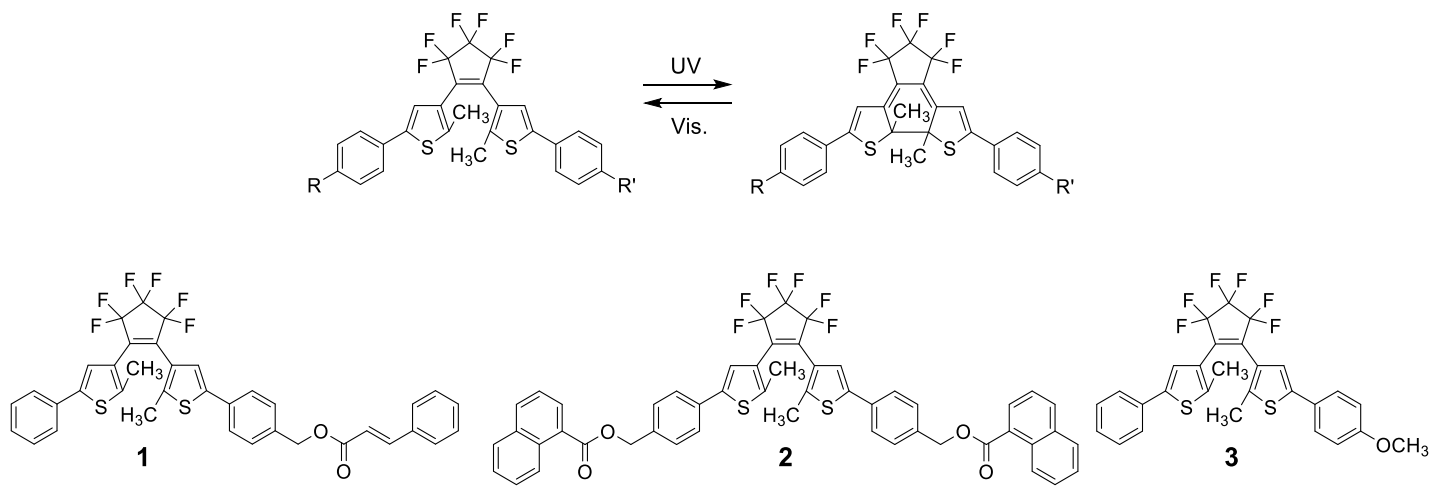

Scheme 1. Diarylethene derivatives used in this work: (1) bending toward UV light; $(\mathbf{2}, \mathbf{3})$ bending away from UV light.

(a)

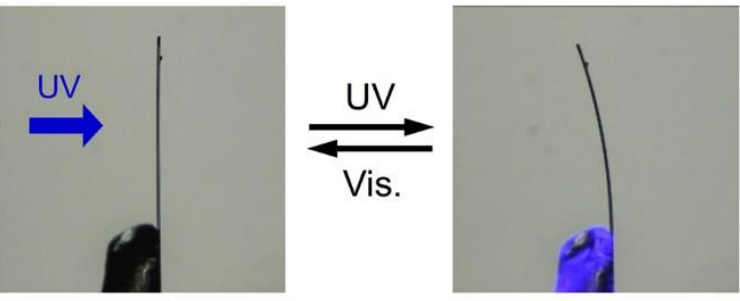

(b)

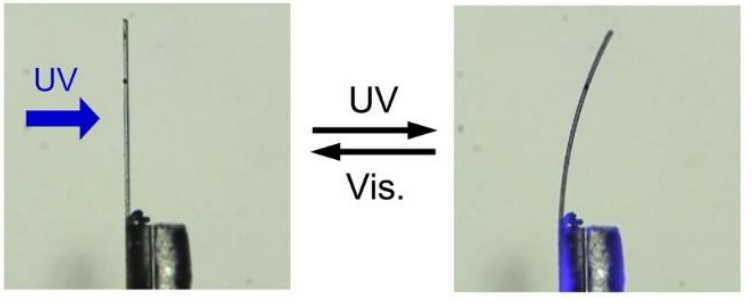

(c)

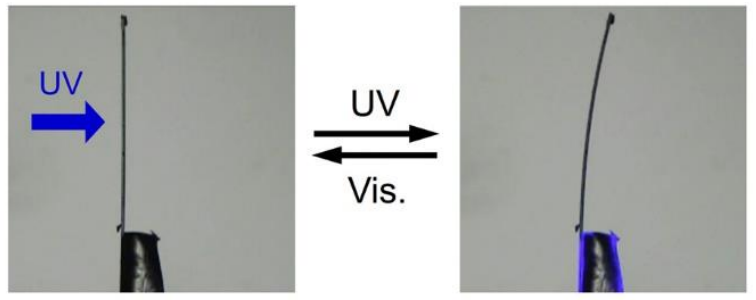

Figure 1. Photoinduced bending behavior of diarylethenes (a) 1, (b) 2, and (c) 3 . 

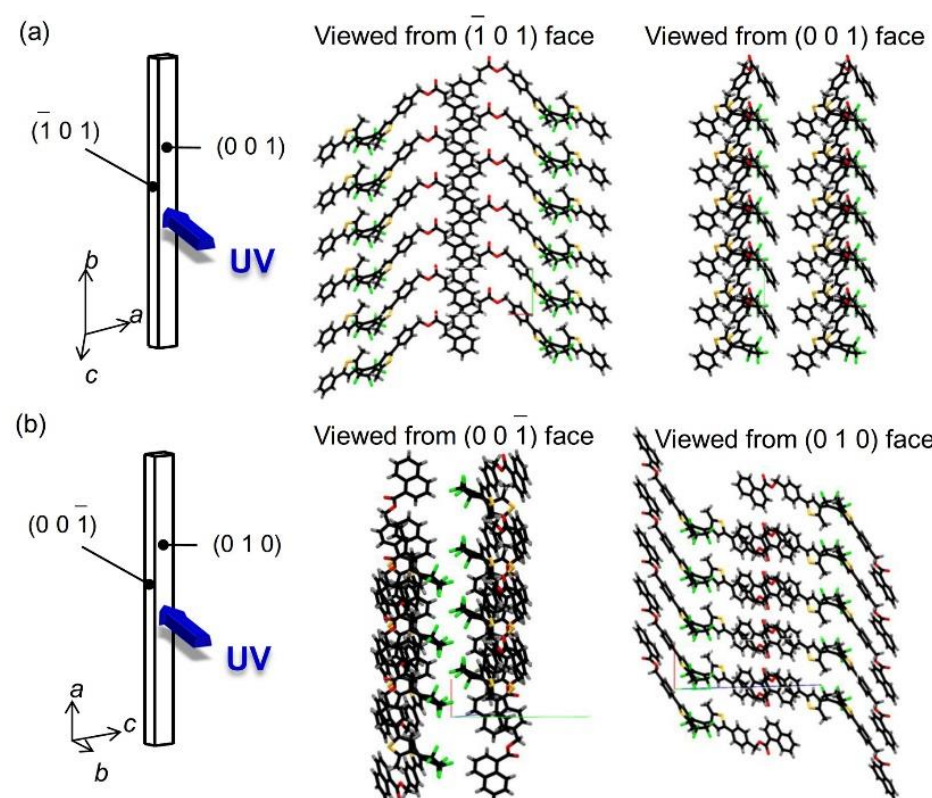

Viewed from $(00 \overline{1})$ face
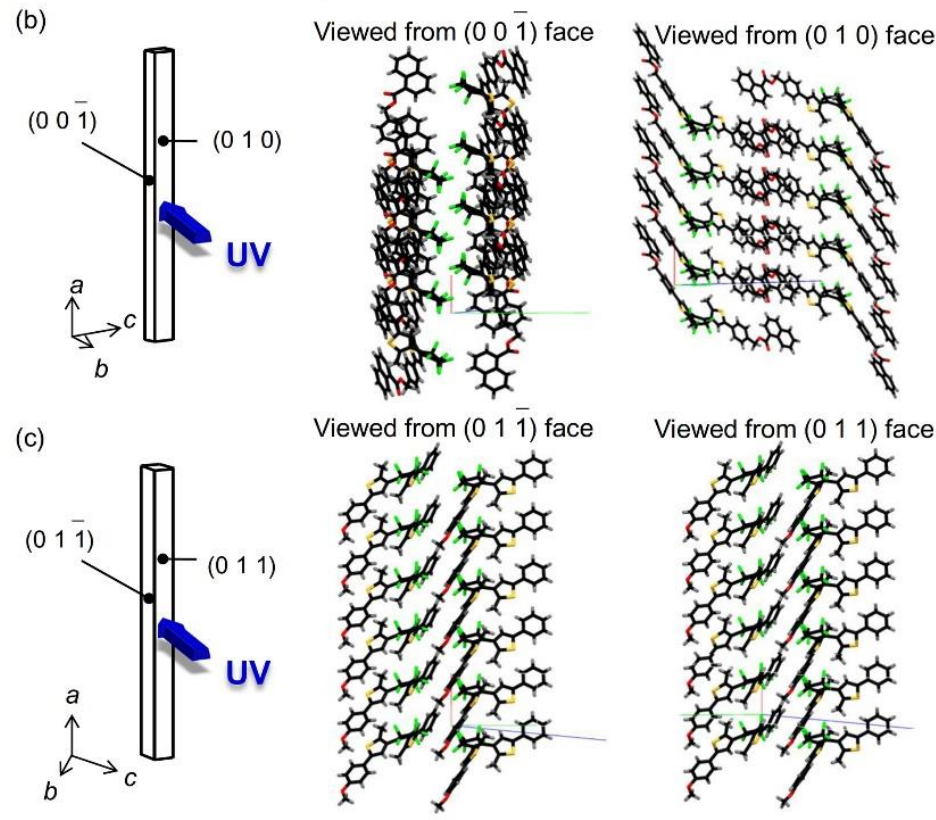

Figure 2. Crystal shapes and molecular packing diagrams of crystals (a) 1, (b) 2, and (c) 3. 

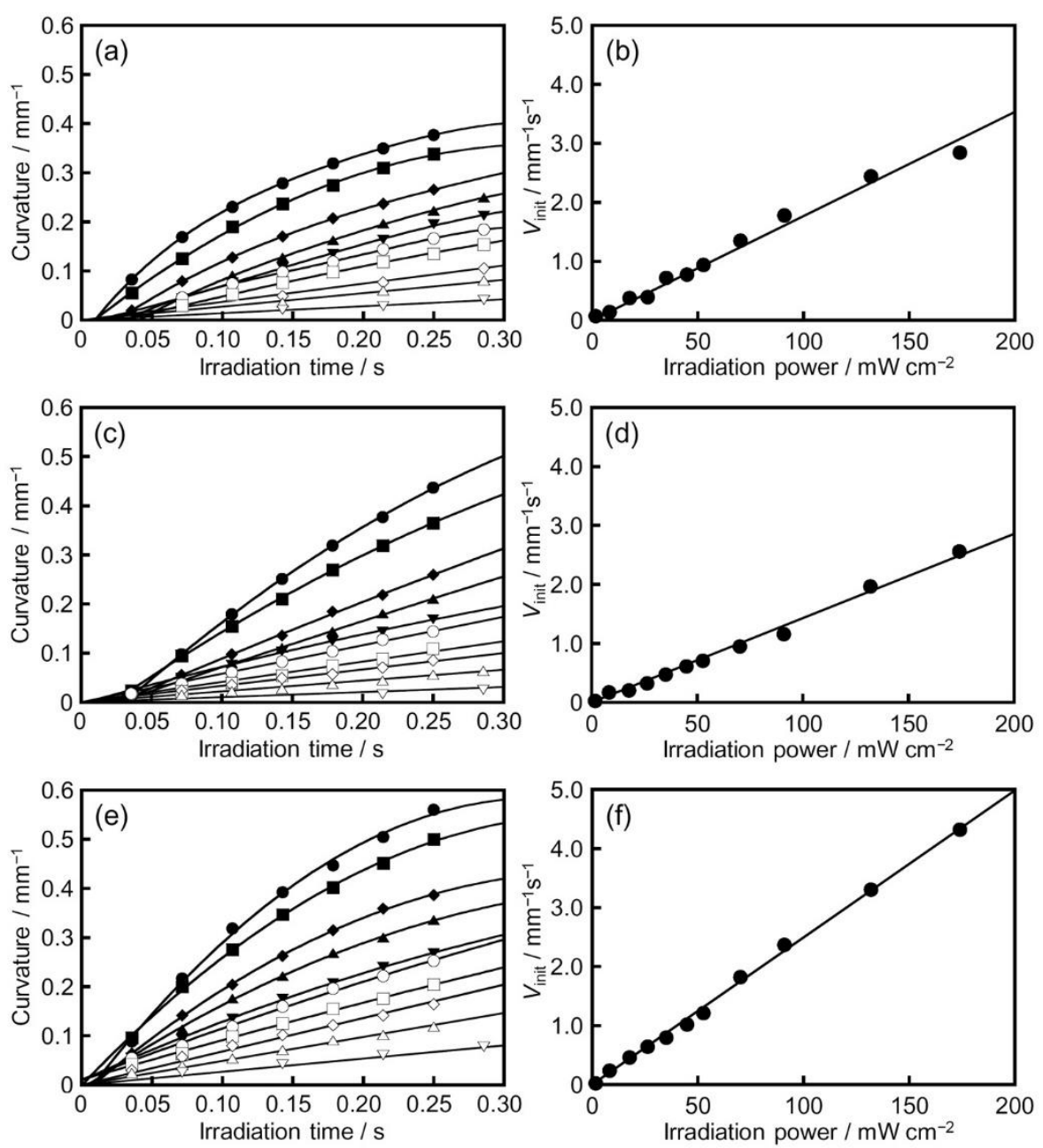

Figure 3. Curvature change as a function of UV irradiation time (a, c, e) and the initial velocity of curvature change $\left(V_{\text {init }}\right)$ as a function of the power of the incident UV light $(b, d, f)$ for crystals $\mathbf{1}$ (a, b; thickness: $7.3 \mu \mathrm{m}), \mathbf{2}$ (c, d; thickness: $6.7 \mu \mathrm{m}$ ), and $\mathbf{3}$ (e, f; thickness: $2.7 \mu \mathrm{m}$ ). The power of the incident UV light was $174(\boldsymbol{O}), 132(\boldsymbol{\nabla}), 91.0(\bullet), 70.2(\boldsymbol{\Delta}), 52.7(\boldsymbol{\nabla}), 44.8(\bigcirc)$, 35.1 ( $\square), 26.3(\diamond), 17.6(\triangle)$, and $8.17 \mathrm{~mW} \mathrm{~cm}^{-2}(\nabla)$ for (a), (c), and (e). 

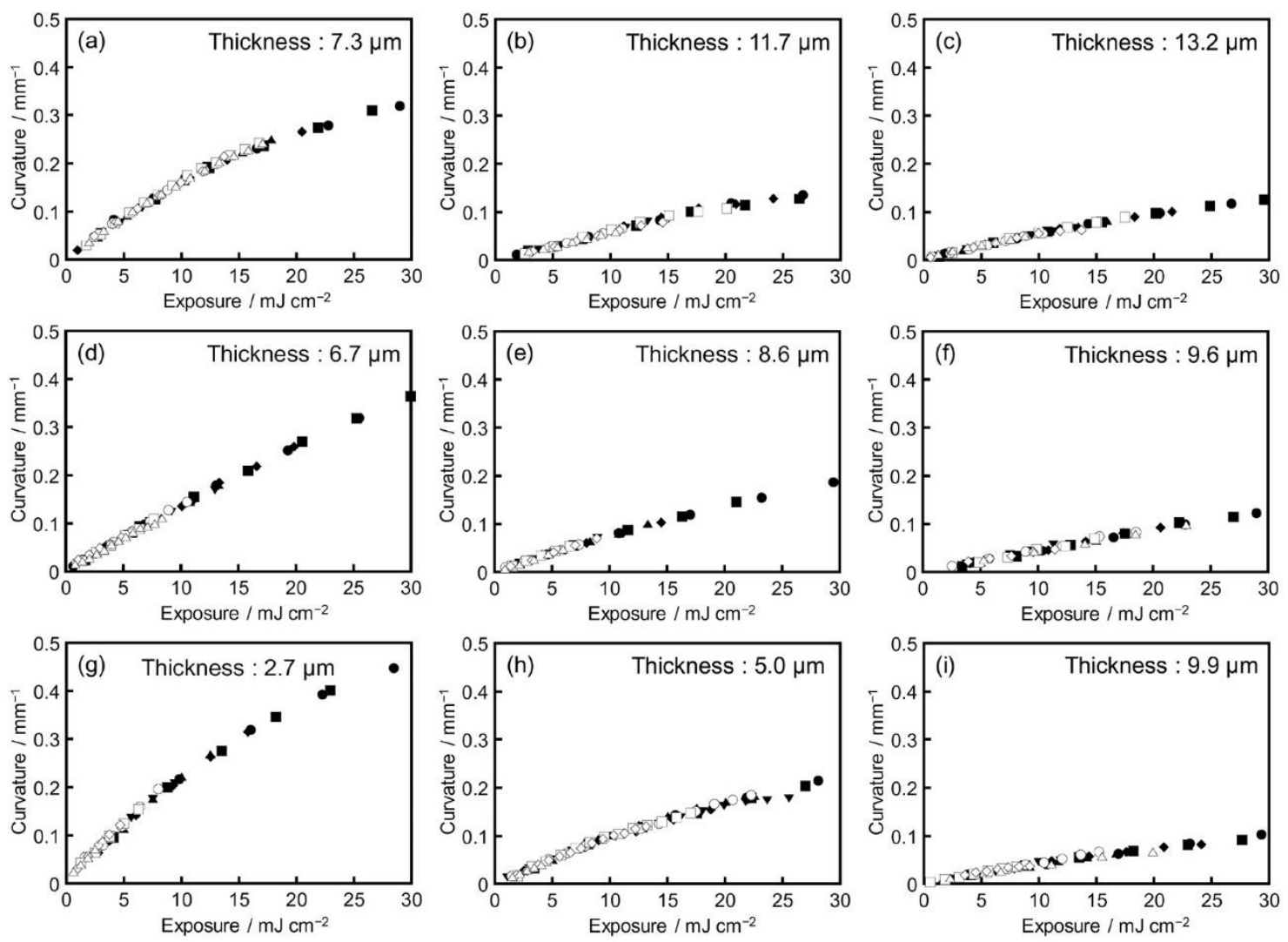

Figure 4. Curvature change as a function of the incident UV exposure (= multiplication of irradiation time and power of light) for crystals 1 (a-c), 2 (d-f), and 3 (g-i). The power of the incident UV light was $174(\boldsymbol{O}), 132(\boldsymbol{\square}), 91.0(\bullet)$ ), $70.2(\boldsymbol{\Delta}), 52.7(\boldsymbol{\nabla}), 44.8(\bigcirc), 35.1$ $(\square), 26.3(\diamond), 17.6(\triangle)$, and $8.17 \mathrm{~mW} \mathrm{~cm}^{-2}(\nabla)$. 

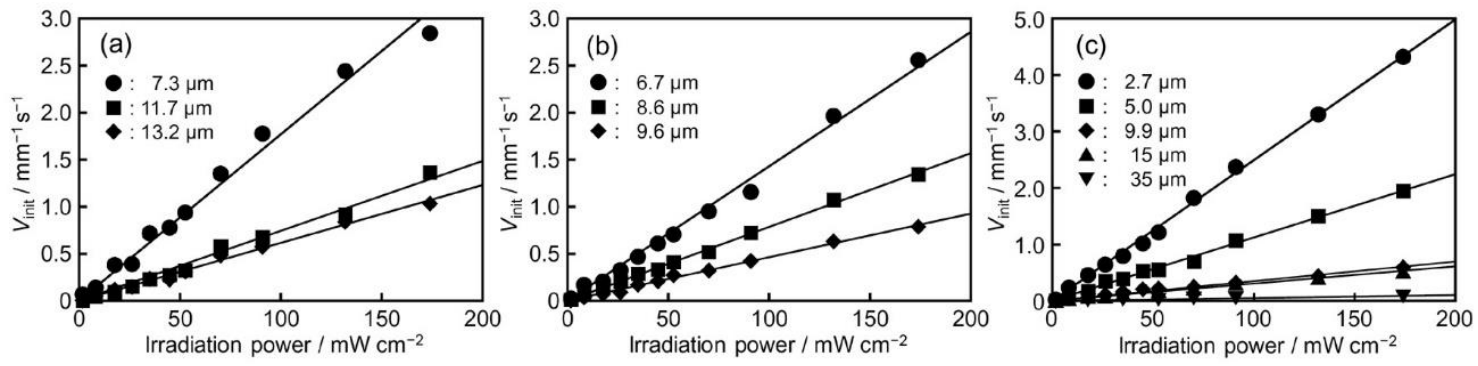

Figure 5. Initial velocity of curvature change $\left(V_{\text {init }}\right)$ as a function of the power of UV light irradiation for diarylethene crystals (a) 1, (b) $\mathbf{2}$, and (c) $\mathbf{3}$ with various crystal thicknesses.

(a) Bending toward UV light

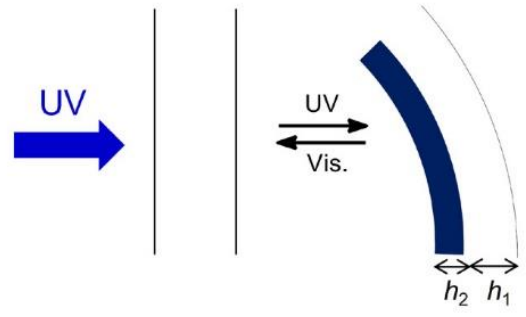

(b) Bending away from UV light

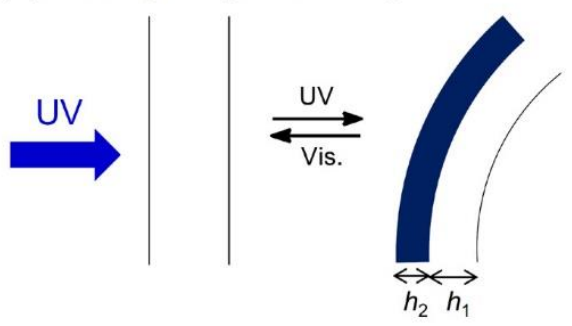

Figure 6. Illustration of the Timoshenko bimetal model in the case of (a) bending toward UV light and (b) bending away from UV light. 

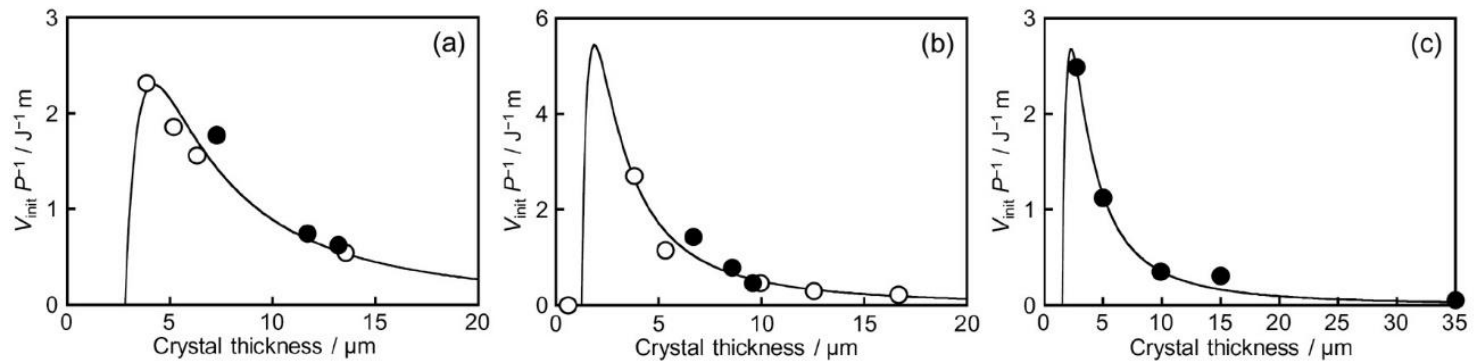

Figure 7. Initial velocity of curvature change relative to the crystal thickness upon irradiation with UV light for diarylethene crystals of $\mathbf{1 - 3}$. The initial velocity is normalized according to the power of the light. The open circles indicate data cited from Ref. 26. The solid lines show fitting curves using the bimetal model with the $h_{2}$ and $\alpha_{2, \text { init }}$ parameters shown in Table 2. 
Table 1. Crystal thickness and $V_{\text {init }} P^{-1}$ for diarylethene crystals 1-3.

\begin{tabular}{|c|c|c|c|c|c|}
\hline \multicolumn{2}{|c|}{1} & \multicolumn{2}{|c|}{2} & \multicolumn{2}{|c|}{3} \\
\hline Thickness & $V_{\text {init }} P^{-1}$ & Thickness & $V_{\text {init }} P^{-1}$ & Thickness & $V_{\text {init }} P^{-1}$ \\
\hline$/ \mu \mathrm{m}$ & $/ \mathrm{J}^{-1} \mathrm{~m}$ & $/ \mu \mathrm{m}$ & $/ \mathrm{J}^{-1} \mathrm{~m}$ & $/ \mu \mathrm{m}$ & $/ \mathrm{J}^{-1} \mathrm{~m}$ \\
\hline $3.88^{\text {a) }}$ & $2.31^{\text {a) }}$ & $0.62^{\text {a) }}$ & $0^{\text {a) }}$ & 2.7 & 2.49 \\
\hline $5.22^{a)}$ & $1.86^{\mathrm{a})}$ & $3.82^{\text {a) }}$ & $2.71^{\text {a) }}$ & 5.0 & 1.12 \\
\hline $6.33^{\text {a) }}$ & $1.56^{\mathrm{a})}$ & $5.34^{\mathrm{a})}$ & $1.15^{\mathrm{a})}$ & 9.9 & 0.348 \\
\hline 7.3 & 1.77 & 6.7 & 1.43 & 15 & 0.305 \\
\hline 11.7 & 0.743 & 8.6 & 0.785 & 35 & 0.0489 \\
\hline 13.2 & 0.615 & 9.6 & 0.464 & & \\
\hline \multirow[t]{3}{*}{$13.56^{a)}$} & $0.536^{a)}$ & $9.97^{\text {a) }}$ & $0.465^{a)}$ & & \\
\hline & & $12.58^{a)}$ & $0.299^{\text {a) }}$ & & \\
\hline & & $16.7^{\text {a) }}$ & $0.221^{\text {a) }}$ & & \\
\hline
\end{tabular}

a) Ref. 26 
Table 2. $h_{2}$ and $\alpha_{2}$,init $P^{-1}$ for diarylethenes 1-3.

\begin{tabular}{cccc}
\hline & $\mathbf{1}$ & $\mathbf{2}$ & $\mathbf{3}$ \\
\hline$h_{2} / \mu \mathrm{m}$ & 2.8 & 1.2 & 1.5 \\
$\alpha_{2, \text { init }} P^{-1} \times 10^{6} / \mathrm{J}^{-1} \mathrm{~m}^{2}$ & & & \\
& 7.3 & & \\
$h_{2} \alpha_{2, \text { init }} P^{-1} \times 10^{12} / \mathrm{J}^{-1} \mathrm{~m}^{3}$ & & & \\
& 21 & 9.5 & \\
\hline
\end{tabular}




\section{For Table of Contents Use Only}

Dependence of Photoinduced Bending Behavior of Diarylethene Crystals on Ultraviolet Irradiation Power

Akira Hirano, Takuya Hashimoto, Daichi Kitagawa, Kenji Kono, and Seiya Kobatake

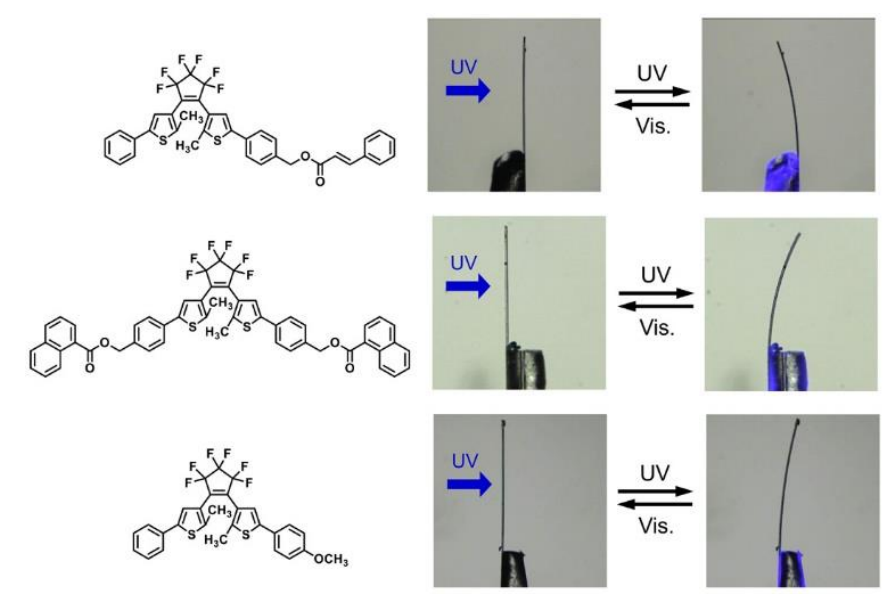

The photoinduced bending behavior of diarylethene crystals upon irradiation with various UV light intensities was investigated. The initial velocity of the photoinduced bending increased in proportion to the power of the incident UV light, even when the crystal thickness was different. This result suggests that the local strain caused by photoisomerization contributes cumulatively to the bending behavior. 